\title{
About a rare case of lupus panniculitis of difficult diagnosis at the Borgou/Alibori Departmental UHC (Benin)
}

\section{Christiane Koudoukpo', Christelle Ahomadégbé ${ }^{2}$, Marie-Claire Ballé1, Fabrice Akpadjan", Bérénice Dégboé ${ }^{3}$, Romulus Takin², Nadège Agbéssi ${ }^{1}$, Luc Brun ${ }^{1}$, Hugues Adégbidi ${ }^{3}$, Félix Atadokpèdé ${ }^{3}$}

${ }^{1}$ Department of Dermatology, University and Departmental Hospital Center of Borgou/Alibori, Parakou, Bénin, ${ }^{2}$ Cancer Oncology Center of Cotonou, Unit of Pathological Anatomy and Cytology, Cotonou, Bénin, ${ }^{3}$ Centre National Hospitalier Universitaire - Hubert Koutoukou, Cotonou, Bénin

Corresponding author: Dr. Christiane Koudoukpo, E-mail: chkoudoukpo@yahoo.fr

\begin{abstract}
We report an unusual case of lupus panniculitis or Kaposi-Irgang disease, of difficult diagnosis. A 40-year-old woman without particular pathological history had consulted in the Service of Dermatology of the Departemental CHU Borgou/Alibori for nodular and painful lesions of the trunk and the thoracic members having begun in the right breast and evolved for eight continuous months. The dermatological examination discovered on the trunk and the thoracic members, nodular hurts under an erythema skin, painful at the palpation; of diameters varying between one and five centimeters. Anatomical examination and immunohistochemistry helped to retain the diagnosis of lupus panniculitis and the patient was given Prednisone orally in degressive doses. Skin lesions regressed after three months. Anatomicalclinical confrontation is essential to retain a positive diagnosis of rare skin diseases such as panniculitis lupus.
\end{abstract}

Key words: Lupus panniculitis; Deep biopsy; Lymphocytic infiltrate; Benin

How to cite this article: Koudoukpo $\mathrm{C}$, Ahomadégbé $\mathrm{C}$, Ballé $\mathrm{M}-\mathrm{C}$, Akpadjan $\mathrm{F}$, Dégboé $\mathrm{B}$, Takin $\mathrm{R}$, Agbéssi N, Brun L, Adégbidi H, Atadokpèdé $\mathrm{F}$. About a rare case of lupus panniculitis of difficult diagnosis at the Borgou/Alibori Departmental UHC (Benin). Our Dermatol Online. 2020;11(1):68-72.

Submission: 10.06.2019; Acceptance: 19.07.2019

DOI: 10.7241/ourd.20201.18 


\title{
A propos d'un cas rare de panniculite lupique de diagnostic difficile au CHU Departemental Borgou/Alibori (Benin)
}

\section{Christiane Koudoukpo', Christelle Ahomadégbé2, Marie-Claire Ballé1, Fabrice Akpadjan ${ }^{3}$,

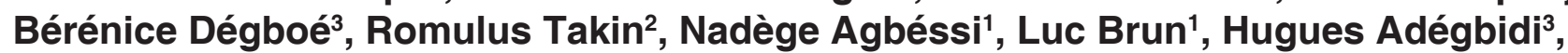 Félix Atadokpèdés}

\begin{abstract}
${ }^{1}$ Department of Dermatology, University and Departmental Hospital Center of Borgou/Alibori, Parakou, Bénin, ${ }^{2}$ Cancer Oncology Center of Cotonou, Unit of Pathological Anatomy and Cytology, Cotonou, Bénin, ${ }^{3}$ Centre National Hospitalier Universitaire - Hubert Koutoukou, Cotonou, Bénin
\end{abstract}

Corresponding author: Dr. Christiane Koudoukpo, E-mail: chkoudoukpo@yahoo.fr

\begin{abstract}
RÉSUMÉ
Nous rapportons un cas inhabituel de panniculite lupique ou maladie de Kaposi-Irgang, de diagnostic difficile. Une femme âgée de 40 ans sans antécédent pathologique particulier avait consulté dans le Service de Dermatologie du CHU Départemental Borgou/Alibori à Parakou pour des lésions nodulaires douloureuses du tronc et des membres thoraciques ayant débuté au sein droit depuis huit mois sur un mode continu. Lexamen dermatologique découvrait sur le tronc et les membres thoraciques, des lésions nodulaires sous une peau érythémateuse, douloureuses à la palpation; les diamètres de ces lésions variaient entre un et cinq centimètres. Lexamen anatomopathologique et l'immunohistochimie ont permis de retenir le diagnostic de panniculite lupique et la patiente a été soumise à la Prednisone par voie orale, à doses dégressives. Les lésions cutanées ont régressé au bout de trois mois. Des conseils diététiques liés à une corticothérapie au long cours ont été prodigués. Une confrontation anatomo-clinique est indispensable pour retenir un diagnostic positif des dermatoses rares comme la panniculite lupique.
\end{abstract}

Mots clés: Panniculite lupique; Biopsie profonde; Infiltrat lymphocytaire; Bénin

\section{INTRODUCTION}

La panniculite lupique ou maladie de Kaposi-Irgang est une forme clinique rare du lupus érythémateux chronique $[1,2]$. La panniculite est constatée dans plusieurs maladies infectieuses, vasculaires, systémiques, de cause physique, pancréatique, ou par déficit en l-antitrypsine, et rarement dans le lupus où elle représente 1 à $3 \%[1,3]$. La panniculite lupique est considérée de nos jours comme un état précancéreux du lymphome T pléomorphe hypodermique [4]. Nous rapportons le cas d'une patiente atteinte de panniculite lupique dont l'examen anatomo-pathologique, en l'occurrence l'étude immunohistochimique a été d'un grand apport au diagnostic qui peut être erroné sans ces outils car la présentation clinique n'est pas la plus spécifique de la maladie lupique.

\section{OBSERVATION}

Une femme âgée de 40 ans sans antécédent pathologique particulier, avait consulté le 15 mai 2018 dans le Service de Dermatologie du CHU Départemental Borgou/Alibori à Parakou (Bénin) pour des lésions nodulaires du tronc et des membres thoraciques. A l'anamnèse, ces lésions non prurigineuses, auraient débuté au sein droit il y a 8 mois, sur un mode continu. Il s'agirait de lésions ponctiformes localisées au début sur le sein gauche; elles auraient augmenté de taille puis étendues progressivement au tronc et aux membres thoraciques. A l'inspection, les

\footnotetext{
How to cite this article: Koudoukpo $\mathrm{C}$, Ahomadégbé $\mathrm{C}$, Ballé M-C, Akpadjan F, Dégboé B, Takin R, Agbéssi N, Brun L, Adégbidi H, Atadokpèdé F. A propos d'un cas rare de panniculite lupique de diagnostic difficile au CHU Départemental Borgou/Alibori (Bénin). Our Dermatol Online. $2020 ; 11$ (1):68-72.

Submission: 10.06.2019; Acceptance: 19.07.2019

DOI: 10.7241 /ourd.20201.18
} 
lésions étaient érythémateuses avec discret phénomène de peau d'orange (Fig. 1). A la palpation, elles étaient douloureuses, sous cutanées et nodulaires, de diamètres variant entre un et cinq centimètres, siégeant sur le tronc et les membres thoraciques (Fig. 2). Les sérologies rétrovirale et hépatique $\mathrm{B}$ et $\mathrm{C}$ étaient négatives. Le bilan immunologique (anticorps anti-ADN natif, anti-Ro/SS-A) était négatif mais le dosage des fractions du complément (C3 et C4) n’a pu être réalisé. Des prélèvements biopsiques cutanés profonds réalisés en zones nodulaires (dos, sein gauche et bras droit) ont révélé:

- au faible grossissement, la présence d'un infiltrat lymphocytaire nodulaire intralobulaire (Fig. 3);

- au fort grossissement des lymphocytes en amas au sein des lobules adipeux, accompagnés de quelques macrophages et d'un foyer de nécrose adipocytaire à type de cytostéatonécrose (Fig. 4).

L'examen anatomopathologique conclut à une hypodermite lymphocytaire nodulaire faisant discuter

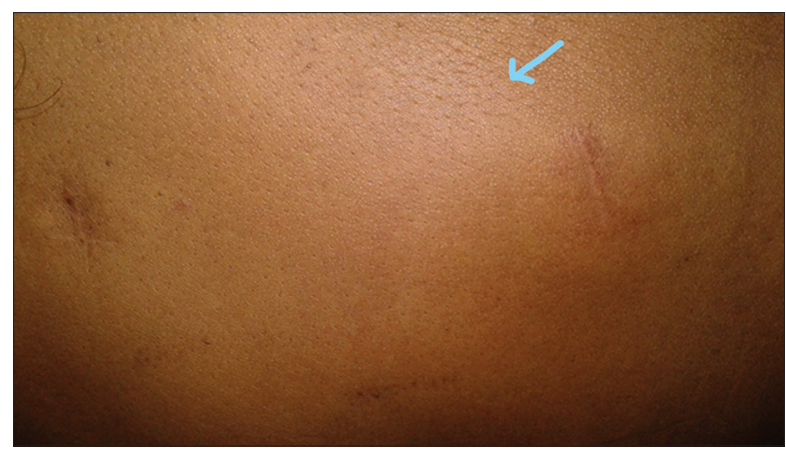

Figure 1: Erythème avec discret phénomène de peau d'orange en regard d'une lésion nodulaire du dos.

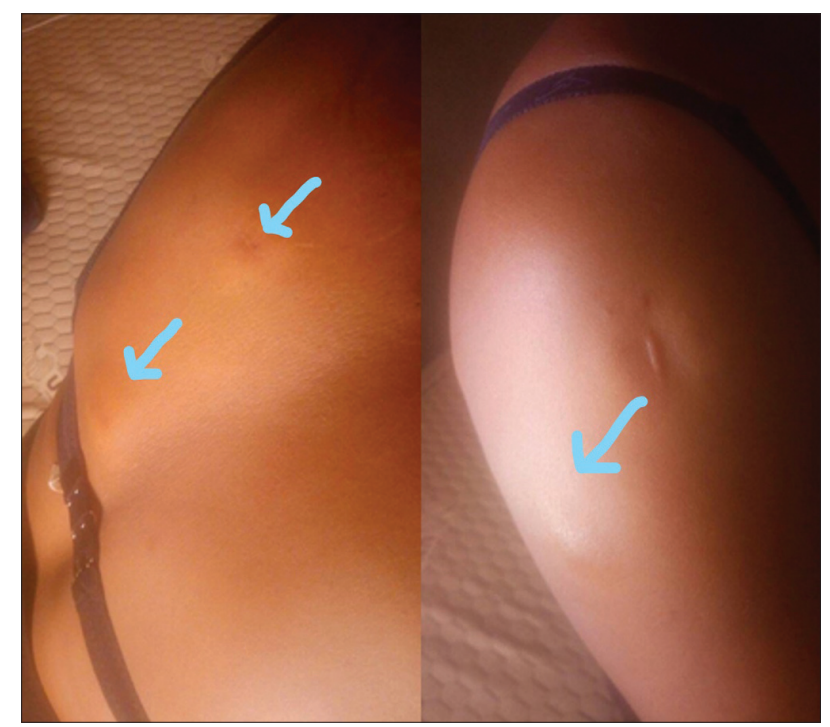

Figures 2: Lésions nodulaires de dimensions $3 \times 5 \mathrm{~cm}$ de grands diamètres du dos et du membre thoracique droit. un lymphome $\mathrm{T}$ panniculite-like ou une panniculite lupique. Létude immunohistochimique a montré des lymphocytes T CD3+/CD8- accompagnés de quelques lymphocytes B réactionnels CD20+ (Fig. 5) permettant d'éliminer le lymphome $\mathrm{T}$ panniculitelike. Le diagnostic de panniculite lupique a été retenu et la patiente a été mise sous Prednisone $1 \mathrm{mg} / \mathrm{kg} /$ jour pendant trois semaines puis à doses dégressives. Les lésions cutanées ont régressé partiellement au bout de 3 mois (Fig. 6). Des conseils diététiques liés à une corticothérapie au long cours ont été prodigués.

\section{DISCUSSION}

La panniculite lupique encore appelée " lupus érythémateux profond " est une forme anatomoclinique rare du lupus érythémateux chronique, souvent associée à un lupus érythémateux systémique dans $10 \%$

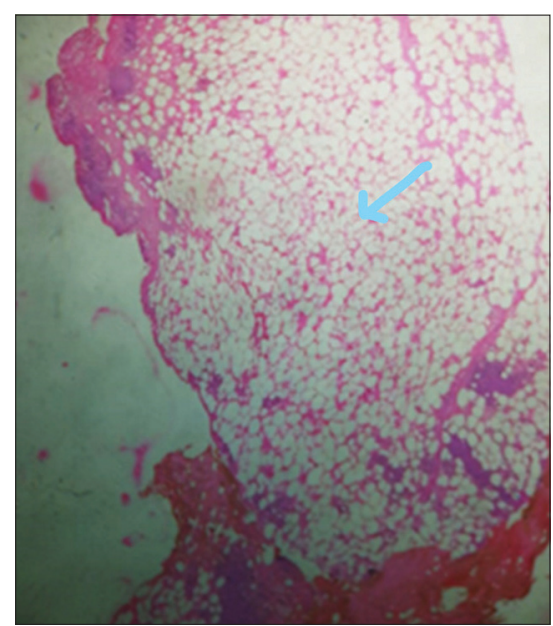

Figure 3: Infiltrat lymphocytaire nodulaire, intralobulaire.

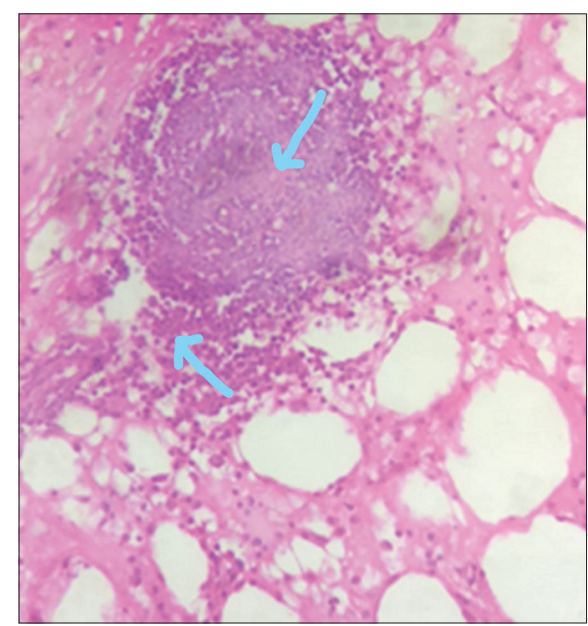

Figure 4: Lymphocytes groupés en amas au sein des lobules adipeux, accompagnés de quelques macrophages et de foyers de nécrose adipocytaire à type de cytostéatonécrose. 


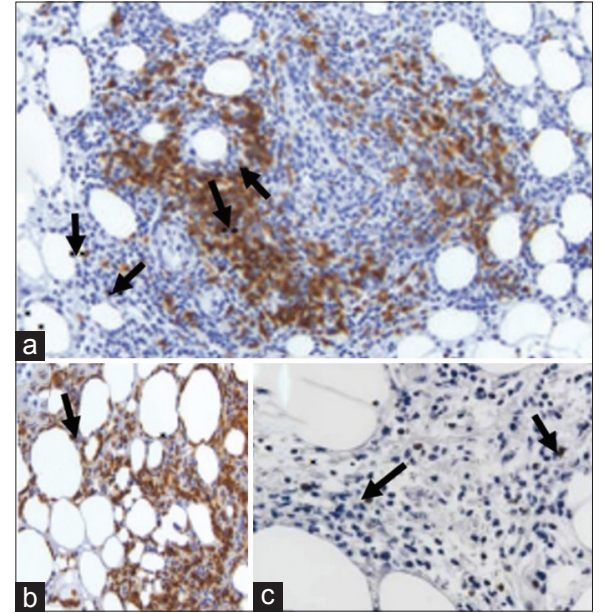

Figures 5:Immunohistochimie: (a) Lymphocytes $B$ de type réactionnel (CD20+); (b) Lymphocytes T CD3+; (c) Lymphocytes T CD8-; x40.

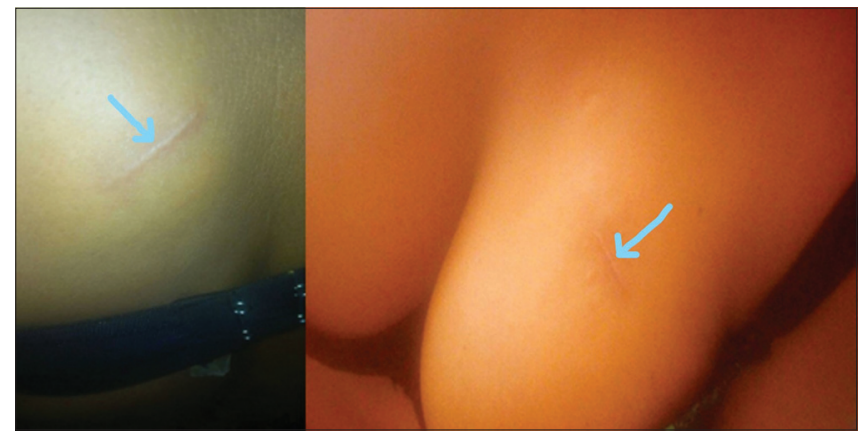

Figures 6: Bonne évolution clinique des lésions au dos et au sein gauche après prélèvements biopsiques et corticothérapie.

des cas $[1,3]$. Le diagnostic repose principalement sur l'intégration des données cliniques et histologiques. Les nodules de panniculite lupique peuvent être isolés ou plus souvent s'intégrer dans une maladie lupique connue, en général de bon pronostic [5,6]. Dans le lymphome, des signes généraux sont plutôt associés tels que la fièvre, les frissons, l'asthénie et l'amaigrissement dans plus de $50 \%$ des cas et la présence dans $25 \%$ des cas, d'adénopathies et/ou d'hépato-splénomégalie [7,8]. Sur le plan biologique, les examens biologiques les plus importants sont immunologiques avec l'identification globale d'anticorps antinucléaires, de ses composantes (anti-ADN natif, anti-Ro/SS-A et La/SS-B, Sm, RNP) et éventuellement la recherche d'une activation du système du complément ainsi qu'une recherche d'anticorps antiphospholipides [9,10]. L'examen histologique nécessite une biopsie large et profonde, à lame de bistouri, comprenant l'hypoderme où se situe l'infiltrat intéressant les lobules graisseux, mimant une panniculite lobulaire. Il permet d'éliminer les principaux diagnostics différentiels que sont le lymphome $\mathrm{T}$ panniculite-like et la vascularite urticarienne [11,12]. On observe habituellement dans le lupus profond, un épidermotropisme lorsque l'épiderme est représenté mais il peut-être quasiabsent avec atteinte du derme profond, comme dans le lymphome $\mathrm{T}$ panniculite-like, égarant le diagnostic $[13,14]$. Dans le lupus, il existe aussi habituellement des dépôts de mucine, la présence de centres germinatifs réactionnels, de foyers de cellules B ou de nombreux plasmocytes, de cellules dendritiques plasmocytoïdes ainsi que de cellules $\mathrm{MxA}$, la présence d'une bande lupique en immunofluorescence directe (IFD) cutanée et l'absence de réarrangement clonal du T-cell receptor (TCR) diagnostic $[15,16]$.

Létude immunohistochimique permet en général de lever l'équivoque. Dans la maladie lupique, comme dans notre cas, les lymphocytes T sont de phénotype CD3+/ CD8-. Dans les lymphomes, ils sont de phénotypes CD3+/CD8+/CD56-/CD30- TCR alpha-bêta avec expression des protéines cytotoxiques comme le granyme B, et présence d'un trou phénotypique CD2, CD5 et/ou CD7 [17,18].

La difficulté diagnostique reste toutefois accrue par l'existence d'authentiques associations entre le lupus et le lymphome, et par la possible coexistence d'aspects histologiques des deux sur différents champs du même prélèvement biopsique [19].

Certains auteurs $[19,20]$ suggèrent que ces 2 pathologies appartiendraient à un même spectre. Le diagnostic de lymphome doit être envisagé devant un lupus profond et, inversement, qu'un lupus systémique soit recherché dans la surveillance des patients atteints de lymphome sous-cutané et panniculite-like. Lévolution clinique du lupus profond se fait vers le développement de cicatrices dystrophiques inesthétiques malgré un traitement bien conduit. Lautre hantise reste, comme soulignés par certains auteurs [19,20], une évolution de la maladie lupique vers le lymphome.

\section{CONCLUSION}

La panniculite lupique est inhabituelle, de diagnostic parfois difficile avec le lymphome qui est en l'occurrence, aussi bien un diagnostic différentiel, qu'une phase évolutive de la maladie. Une bonne clinique avec des tests immunologiques adéquats et une biopsie cutanée profonde et large avec une étude immunohistochimique permettent de faire un diagnostic de précision. Lévolution est souvent 
favorable mais le challenge reste dans une surveillance rigoureuse de ces sujets à risque de développer un lymphome.

\section{Consent}

The examination of the patient was conducted according to the Declaration of Helsinki principles.

\section{RÉFÉRENCES}

1. Requena L. Normal subcutaneous fat, necrosis of adipocytes and classification of the panniculitides. Semin Cutan Med Surg. 2007;26:66-70.

2. Francès $\mathrm{C}$, Barète $\mathrm{S}$, Piette J. Dermatologic manifestations in lupus erythematosus. Rev Med Interne. 2008;29:701-9.

3. Jourdan M, Chably G, Bagot M, Chatelain D, Lok C. Panniculite lupique de diagnostic difficile. Ann Dermatol Venereol. 2011;138(12S):265-6.

4. Boudhas A, Allaoui M, Reda El Ochi M, Oukabli M, Al Bouzidi A. Une cause rare de panniculite: le lymphome T sous-cutané. Research Fr. 2017;4:2399.

5. Ely SO, Nacerdine B, Abdou J. Lymphome T cutané périphérique pléomorphe CD4+. Ann Dermatol Venereol. 2016;143:62.

6. Cribier B. Panniculite lupique. La Presse Médicale. 2005;34:243-8.

7. Lozzi GP, Massone C, Citarella L, Kerl H, Cerroni L. Rimming of adipocytes by neoplastic lymphocytes: a histopathologic feature not restricted to subcutaneous T-cell lymphoma. Am J Dermatopathol. 2006;28:9-12.

8. Feugier P, Delarue R, de Kerviler E, Gallais JL. Perceptions et pratiques des médecins francais pour initier le diagnostic du lymphome en 2009: première enquête nationale IDiL. Correspondan Onco-Hématol. 2010;1:42-8.

9. Vasquez-Canizares N, Wahezi D, Putterman C. Diagnostic and prognostic tests in systemic lupus erythematosus. Best Pract Res Clin Rheumatol. 2017;31:351-63.

10. Goetz J. Marqueurs biologiques anciens et modernes du lupus érythémateux systémique. Rev Rhumatis. 2005;73:134-41.

11. Francès C. Lupus érythémateux disséminé. Syndrome des antiphospholipides. Ann Dermatol Venereol. 2002;129:2S102-5.

12. Takeshita M, Imayama S, Oshiro Y, Kurihara K, Okamoto S, Matsuki Y, et al. Clinicopathologic analysis of 22 cases of subcutaneous panniculitis-like CD56- or CD56+ lymphoma and review of 44 other reported cases. Am J Clin Pathol. 2004;121:408-16.

13. Franck N, Fraitag S. Lymphome T sous-cutané à type de panniculite. Correspondan Onco-Hématol. 2015;10:60-4.

14. Cotin Bordes C, Lazaro E, Pellegrin J-L, Viallard J-F, Moreau J-F, et al. Lupus érythémateux systémique: de la physiopathologie au traitement. Rev Med Interne. 2009;30:9-13.

15. Massone C, Kodama K, Salmhofer W, Abe R, Shimizu H, Parodi A, et al. Lupus erythematosus panniculitis (lupus profundus): clinical, histopathological, and molecular analysis of nine cases. J Cutan Pathol. 2005;32:396-404.

16. Gill MA, Bajwa G, George TA, Dong CC, Dougherty II, Jiang N, et al. Counterregulation between the FcepsilonRI pathway and antiviral responses in human plasmacytoid dendritic cells. J Immunol. 2010;184:5999-6006.

17. Willemze R, Jansen PM, Cerroni L, Berti E, Santucci M, Assaf C, et al. Subcutaneous panniculitis-like T-cell lymphoma: definition, classification, and prognostic factors: an EORTC Cutaneous Lymphoma Group Study of 83 cases. Blood. 2008;111:838-45.

18. Bagot M, Bensussan A. Les lymphomes T épidermotropes comme modèles de progression tumorale. Med Sci. 2006;22:192-6.

19. Bosisio F, Boi S, Caputo V, Chiarelli C, Oliver F, Ricci R, et al. Lobular panniculitis infiltrates with overlapping histopathologic features of lupus panniculitis (lupus profundus) and subcutaneous T-cell lymphoma: a conceptual and practical dilemma. Am J Surg Pathol. 2015;39:206-11.

20. Delsol G. The 2008 WHO lymphoma classification. Ann Pathol. 2008;120-4.

Copyright by Christiane Koudoukpo, et al. This is an open-access article distributed under the terms of the Creative Commons Attribution License, which permits unrestricted use, distribution, and reproduction in any medium, provided the original author and source are credited.

Source of Support: Nil, Conflict of Interest: None declared. 